\title{
Pérdida de peso seco en hojarasca de Alnus acuminata en la provincia de Tucumán (Argentina)
}

\author{
Dry weight loss of litter of Alnus acuminata in the province of Tucumán, Argentina
}

C.D.O.: 114.35

\author{
PABLO G. ACEÑOLAZA ${ }^{1}$, JUAN F. GALLARDO-LANCHO ${ }^{2}$ \\ ${ }^{1}$ Facultad de Ciencias Naturales e Instituto Miguel Lillo, U.N.T., San Miguel de Tucumán 4000 (R. Argentina). \\ ${ }^{2}$ C.S.I.C., Aptdo. 257, Salamanca 37071, España.
}

\begin{abstract}
SUMMARY
The decomposition rate of the leaves of Alnus acuminata H.B.K spp. acuminata, was studied during a two year period using the litter bag procedure. The experiment was performed in forest stands of three age group (5-10, 2025 and 40-45 yr) located in San Javier hills, Province of Tucumán (Argentina). A regression between the remnant percentage of dry matter and time was carried out adjusting the curve to an exponential model. The pattern of decomposition presented the most important loss of weight during the first 30 days, the rate decreasing slowly later on. Highly significant differences were observed because of the canopy closure or because the differences between the leaf chemical composition of the different age of the stands or because of both. Results are similar to those obtained by other authors working on the same genera.
\end{abstract}

\section{RESUMEN}

Se estudió la dinámica de descomposición de hojas de Alnus acuminata H.B.K. spp acuminata en tres bosques de esta especie de distintas edades (5-10, 20-25 y 40-45 años) situados en el cerro de San Javier (Tucumán, Rep. Argentina), durante el período abril/'91 a abril/'93, siguiendo la metodología de las "litter bags". Se realizó una regresión entre los valores de porcentaje de materia seca remanente y el tiempo, ajustándose la curva de descomposición a un modelo exponencial. En este patrón de descomposición se observa una rápida pérdida de peso durante los 30 primeros días, decayendo posteriormente el índice de descomposición. Se obtuvieron diferencias altamente significativas en los índices de descomposición del bosque de 20-25 años de edad con respecto a los otros dos bosques, pudiendo deberse bien al cerramiento del dosel arbóreo y/o a la diferente composición de las hojas a distintas edades de los bosques. Los índices de descomposición obtenidos son del mismo orden que los citados por otros autores para otras especies de este género botánico.

\section{INTRODUCCION}

Entre los numerosos aspectos que se han venido abordando durante los últimos años, cabe citar los referentes a la dinámica de descomposición de la hojarasca y consecuente liberación de bioelementos (Vilas Boas et al., 1993). Esta hojarasca sufre transformaciones catabólicas más o menos intensas que, por un lado, originan liberaciones de nutrientes y, por otro, forman sustancias estables y oscuras denominadas húmicas, que contribuyen a la capacidad de cambio del suelo y al mejoramiento de la estructura edáfica (Santa Regina, 1987). En este proceso de descomposición actúan numerosos microorganismos heterótrofos (bacterias y hongos), por lo cual gran parte de los factores que regulan la actividad de éstos inciden a su vez en los procesos de humificación/descomposición (Jansson y Berg, 1985), tal como la temperatura y la humedad, aunque también tiene singular importancia la naturaleza del material orgánico en descomposición (Daubenmire y Prusso, 1963; Dyer et al., 1992).

El aliso andino (Alnus acuminata H.B.K. spp. 
acuminata) es la única especie de la familia Betulacea en el Hemisferio Sur. En la provincia de Tucumán (Argentina) llega a formar bosques continuos en laderas húmedas situadas entre los 1.500 y 2.500 m s.n.m. (Aceñolaza et al., 1992).

Consecuentemente, el objetivo del presente trabajo es conocer el índice de descomposición de las hojas de aliso en tres bosques de diferentes edades de la provincia de Tucumán (Rep. Argentina), y las relaciones de aquel parámetro con los factores abióticos ambientales. Por tanto, se desea establecer si los índices de descomposición de las hojas de alisos varían con la edad del bosque o no; de otra manera, si las condiciones microambientales, y/o la diferente composición de la hojarasca (si la hubiere), causada por el desarrollo del bosque (aliseda), produce un cambio significativo en la velocidad de descomposición de la hojarasca.

\section{MATERIAL Y METODOS}

El presente estudio se llevó a cabo en alisedas del Parque Biológico de la Sierra de San Javier (reserva natural de la Universidad Nacional de Tucumán). El clima del área considerada es húmedo-templado (húmedo mesotérmico), que según el sistema climático de Köppen corresponde a la fórmula Cwb (Aceñolaza et al., 1992).

Se eligieron tres alisedas de diferentes edades: una "joven" (5 a 10 años), otra que se denomina "madura" (20 a 25 años) y una última considerada "vieja" (40 a 50 años), procurando que se encuentren cercanas unas a otras y en situaciones geomorfológicas similares.

Para seguir la evolución de la descomposición de las hojas se confeccionaron bolsas (litter bags) en las que se introdujo un peso conocido de hojas de aliso; este método ha sido utilizado con éxito por diferentes investigadores (Bocock et al., 1960; Edmonds, 1979; Sharma y Ambasht, 1987), teniendo siempre en cuenta las limitaciones que el mismo posee, entre éstas, la inaccesibilidad de la microfauna (Santa Regina 1987; Hernández et al., 1992). Las bolsas de $18 \times 15 \mathrm{~cm}$ se fabricaron con malla de nylon de $1 \mathrm{~mm}$ de luz; en cada una de ellas se introdujeron exactamente $10 \mathrm{~g}$ de hojas (peso fresco, humedad conocida) de aliso cercanas a la abscisión. Se colocaron 50 bolsas bajo los árboles en cada una de las alisedas citadas, procurando que todas las bolsas permanecieran en condiciones ecológicamente semejantes. Durante dos años se fueron retirando progresivamente 5 bolsas por bosque, desde abril de 1991 hasta abril de 1993 (a $\operatorname{los} 2,6,14,30,62,126,254$ y 715 días desde el inicio) (Vilas Boas et al., 1993). Posteriormente los residuos de las hojas se limpiaron cuidadosamente con aire, se secaron a $70^{\circ} \mathrm{C} \mathrm{du}$ rante $48 \mathrm{~h}$ y se pesaron en balanza de precisión.

Para establecer la relación entre el residuo de hojas y el tiempo de la experiencia se realizó una regresión, considerando como variable independiente el tiempo (días), aplicando posteriormente un ANOVA a los resultados obtenidos en cada uno de los bosques (Wieder y Lang 1982; Hernández et al., 1992).

\section{RESULTADOS Y DISCUSION}

En el cuadro 1 se presentan los valores medios y sus desviaciones estándares (SD) de peso seco de hojas (g) en diferentes fechas y para cada una de las alisedas citadas.

\section{CUADRO 1}

Valores medios $(n=5)$ de peso seco remanente de hojas (g) a lo largo del tiempo (días), con las desviaciones estándares (SD) correspondientes.

Average rates $(\mathrm{n}=5)$ of renmant dry weight of leaves ( $\mathrm{g}$ ) through time (days) with the corresponding standard

deviations.

\begin{tabular}{|llll|}
\hline Día & Aliseda joven & Aliseda madura & Aliseda vieja \\
\hline 0 & $2.96(0.29)$ & $2.85(0.12)$ & $2.74(0.04)$ \\
2 & $2.74(0.27)$ & $2.73(0.17)$ & $2.66(0.22)$ \\
6 & $2.67(0.32)$ & $2.40(0.17)$ & $2.57(0.30)$ \\
14 & $2.36(0.29)$ & $2.33(0.14)$ & $2.19(0.21)$ \\
30 & $2.13(0.13)$ & $2.27(0.13)$ & $2.26(0.19)$ \\
62 & $2.15(0.14)$ & $2.32(0.16)$ & $2.39(0.17)$ \\
126 & $2.08(0.16)$ & $1.42(0.17)$ & $1.81(0.15)$ \\
254 & $2.05(0.31)$ & $1.48(0.32)$ & $1.91(0.26)$ \\
715 & $1.61(0.09)$ & $1.26(0.54)$ & $1.91(0.44)$ \\
\hline
\end{tabular}

Los resultados también se exponen de manera gráfica en la figura 1, en la que en ordenadas se expone el tiempo en días y en las abscisas se recoge el remanente de hojas, expresado en \% referente a la cantidad inicial de hojas colocadas en el "litter bag".

Dinámica de la pérdida de peso seco. Para estimar la dinámica de la pérdida de peso seco se ajustaron los valores a un modelo exponencial 
negativo que ha sido aplicado anteriormente por otros autores (Gosz et al., 1973; Hernández et al., 1992). Jewel (1971) propuso el siguiente modelo de ecuación:

$$
\mathrm{Rt}=\mathrm{A}+\mathrm{B} * \exp (-\mathrm{C} * \mathrm{t})
$$

donde Rt es el residuo orgánico en el momento t, A la fracción orgánica más resistente a la biodegradación (asíntota diferente de cero), B la fracción orgánica lábil, $\mathrm{C}$ es la constante de descomposición y t el tiempo de descomposición en días.

Las ecuaciones obtenidas son las siguientes: Aliseda joven $\mathrm{Rt}=68.16+31.19 * \exp (-0.04 *$ t $) \quad \mathrm{R}^{2}=0.87$ Aliseda madura $\mathrm{Rt}=45.04+49.07 * \exp (-0.01 * \mathrm{t}) \mathrm{R}^{2}=0.99$ Aliseda vieja $\mathrm{Rt}=67.03+27.40 * \exp (-0.02 * \mathrm{t}) \quad \mathrm{R}^{2}=0.80$

Se observa (fig. 1) una rápida pérdida de peso seco durante los primeros 30 días, siendo notablemente más lenta la descomposición a partir de los dos meses. Se constatan diferencias significativas $(\mathrm{p}<0.01)$ en la pérdida de peso entre las diferentes alisedas para los primeros 60 días (cuadro 2); obviamente la significancia baja a partir de $\operatorname{los} 60$ días.

\section{CUADRO 2}

Matriz de significancias (Sig.) obtenida en el ANOVA entre las tres alisedas en relación a la descomposición de las hojas $(n=45)$.

Significance matrix (Sig.) obtained with ANOVA among the three alisedas in relation to leaf decomposition.

\begin{tabular}{|lccc|}
\hline Bosques & Aliseda joven & Aliseda madura & Aliseda vieja \\
\hline A. joven & - & Sig.p $<0.01$ & Sig.p $<0.05$ \\
A. madura & Sig.p $<0.01$ & - & Sig.p $<0.01$ \\
A. vieja & Sig.p $<0.05$ & Sig.p $<0.01$ & - \\
\hline
\end{tabular}

Otros autores (Ewel, 1976; Vilas Boas et al., 1993) han encontrado comportamientos semejantes, con una pérdida rápida durante las tres o cinco semanas iniciales; esta pérdida se atribuye a una lixiviación de materias más o menos solubles por agua de lluvia, que se ve favorecida durante el período lluvioso, como este caso. Estas sustancias fácilmente biodegradables estimulan, a su vez, la actividad microbiana; así Witkamp (1966) opina que la presencia de sustancias energéticas en la primera fase de descomposición produce un crecimiento en las poblaciones microbianas, cuya biomasa puede significar una importante propor-
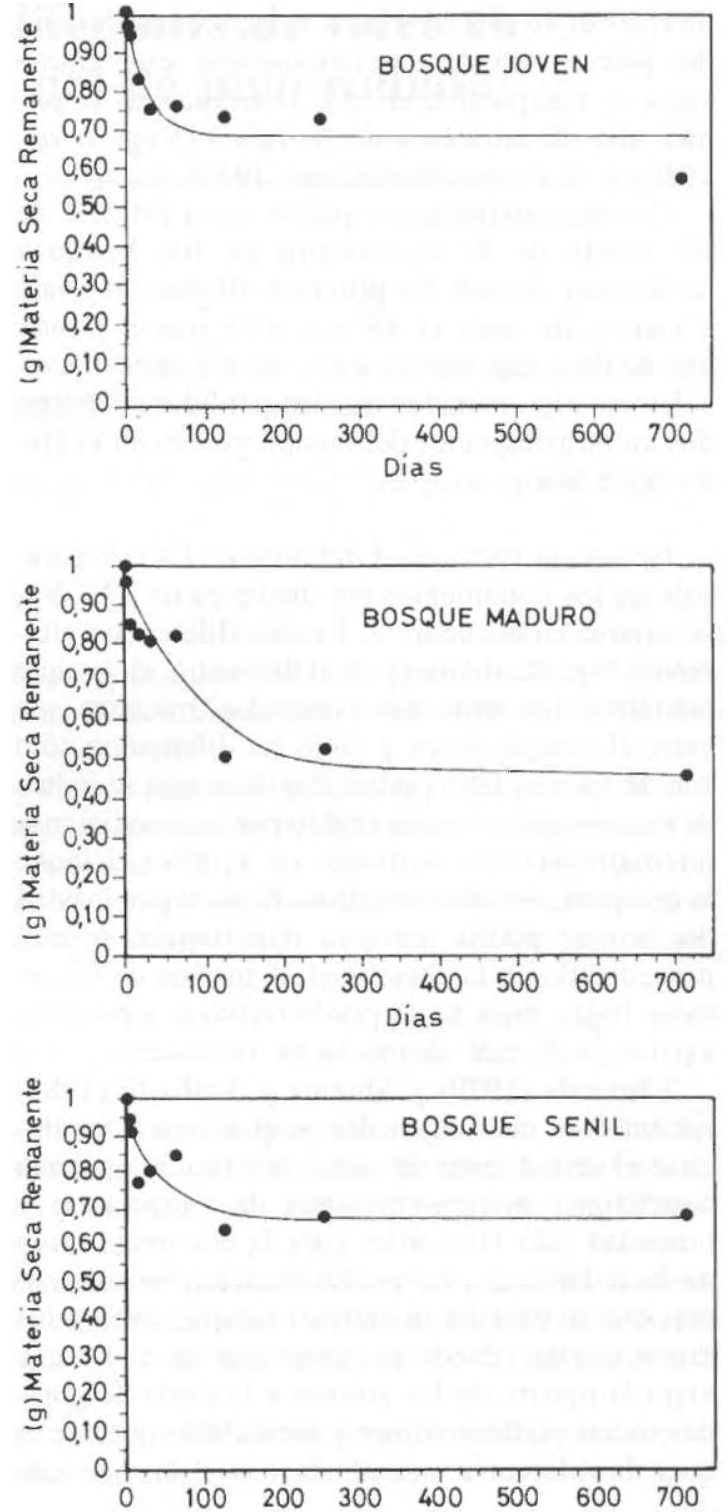

Figura 1. Evolución de la pérdida de peso seco en hojas de aliso en los distintos bosques estudiados.

Evolution of dry weight loss of Alnus acuminata leaves in different studied forest.

ción de peso seco. También Wieder y Lang (1982) aseguran que la principal razón por la que la velocidad de descomposición decrece en el tiempo es que tales sustancias, fáciles de catabolizar, se agotan restando un sustrato más biorresistente. Bocok (1964) encontró una descomposición entre el 70 y $90 \%$ de pérdida de peso seco en $A$. glutinosa en los primeros 8 meses en Inglaterra, mientras que Sharma y Ambasht (1987) encontraron pérdidas 
de entre el 46 y el $61 \%$ para $A$. nepalensis después del primer año de descomposición; este último valor es comparable al $55 \%$ obtenido tras el primer año en hojarasca de A. rubra (Vogt et al., 1980) y A. crispa (Van Cleve, 1971).

Consecuentemente, se pierde cerca del $22 \%$ de las hojas de A. acuminata en los bosques tucumanos durante los primeros 30 días, llegando a valores de entre el 35 y el $55 \%$ tras el primer año de descomposición; estos valores pueden considerarse algo menores que las pérdidas encontradas en otras especies del mismo género en el $\mathrm{He}$ misferio Norte.

Influencia de la edad del bosque. La comparación de los tratamientos por medio de un ANOVA se expuso en el cuadro 2. Existen diferencias altamente significativas $(\mathrm{p}<0.01)$ entre el bosque maduro y los otros dos estudiados, mientras que entre el bosque joven y viejo las diferencias sólo son de un $5 \%$. Esto podría significar que el índice de descomposición está regido por las condiciones microambientales existentes en aquéllos (aunque la composición diferente de las hojas según la edad del bosque podría tener su importancia; se está procediendo en la actualidad al análisis de las citadas hojas, pues no se puede rechazar a priori la existencia de este último factor intrínseco).

Edmonds (1979) y Sharma y Ambasht (1987) encontraron una mayor descomposición al producirse el cerramiento del dosel arbóreo, lo que crea condiciones microambientales de temperatura y humedad más favorables para la descomposición de la hojarasca; esto podría explicar las diferencias que se establecen entre el bosque joven y los dos restantes. Puede pensarse que en el bosque viejo la muerte de los árboles y la caída de grandes ramas pudiera volver a abrir claros que expliquen la diferencia encontrada con el bosque maduro.

Por tanto, del análisis de la dinámica de descomposición de hojarasca de A. acuminata se puede establecer un primer período de pérdida de peso relativamente rápido, que se extiende durante los primeros 30 ó 40 días, y posteriormente un segundo, durante el cual dicha pérdida de materia es progresivamente menor. Las diferencias significativas encontradas en la descomposición de hojas procedentes de alisedas de diferentes edades pueden explicarse por el cerramiento del dosel arbóreo, aunque la posible composición distinta de las hojas pudiera tener también una menor influencia.

\section{AGRADECIMIENTOS}

Al Dr. Gerardo Moreno por su colaboración en el tratamiento de datos.

\section{BIBLIOGRAFIA}

ACEÑOlAZA, P. G., H.R. GRAU, M.F. ARTURI, A.D. BROWN. 1992. "Estructura y dinámica de bosques montanos del noroeste argentino", Actas II Congreso Latinoamericano de Ecología. Caxambú, Brasil (en prensa).

BOCOCK, K.L. 1964. "Changes in the amount of dry matter, N.C. and energy in decomposition woodland leaf litter in relation to the activities of the soil fauna", J. Ecol. 52: 273284.

BOCOCK, K.L., O.J. GILBERT, C.K. CAPSTICK, DC. TURNER, J.S. WARD, M.J. WOODMAN. 1960. "Changes in leaf litter when placed on the surface of soil with contrasting humus type", J. Soil Sci. 11: 1-9.

DAUBENMIRE, R., DC PRUSSO. 1963. "Studies of the decomposition rates of tree litters", Ecology 44: 589-592.

DYER, M.L., V. MEENTEMEYER, B. BERG. 1992. "Apparent controls of mass loss of leaf litter on a regional scale: Litter quality vs. climate", Scand. J. For. Res. 5: $311-$ 323

EDMONDS, R.L. 1979. "Litter decomposition and nutrient release in Douglas-fir needle litter in relation to stand development", Can. J. For. Res. 9: 132-140.

EWEL, J.J. 1976. "Litter fall and leaf decomposition in a tropical forest succession in Eastern Guatemala", J. Ecol. 64: 293-308.

GOSZ, J.R., G.E. LIKENS, F.H. BORMAN. 1973. "Nutrient release from decomposition leaf and branch litter in the Hubbard Brook forest, N.H.", Ecol. Monograph, 43: 173191.

HERNANDEZ, I.M., I. SANTA REGINA, J.F. GALLARDO. 1992. Dinámica de la descomposición de la hojarasca forestal en bosques de la Cuenca del Duero: Modelización de la pérdida de peso. Arid. Soil. Res. \& rehabil., 6: 339-355.

JANSSON, P.E., B. BERG. 1985. "Temporal variation of decomposition in relation to simulated soil climate: Longterm decomposition in a Scots pine forest", Can. J. Bot. 63: 1008-1016.

JEWEL, W.J. 1971. "Aquatic weed decay: dissolved oxygen utilization and $\mathrm{N}$ and $\mathrm{P}$ regeneration", J. Water Pollution Control 43: 1457-1467.

SANTA REGINA, I. 1987. Contribución al estudio de la dinámica de la materia orgánica y bioelementos en bosques de la Sierra de Béjar. Tesis Doctoral. Universidad de Salamanca, 464 pp.

SHARMA E., R.S. AMBASHT. 1987. "Litterfall, decomposition and nutrient release in an age sequence of Alnus nepalensis plantation stands in the Eastern Himalaya", J. Ecol. 75: 997-1010

VAN CLEVE, K. 1971. Energy and weight loss functions or decomposing foliage in birch and aspen forests in Interior Alaska. 52: 720-723.

VILAS BOAS, O., A.C. IMBACH, M.J. MAZZARINO, A. BONNEMANN, J. BEER. 1993. Descomposición de la hojarasca en sistemas agroforestales de Cordial y Erythrina en Turrialba, Costa Rica. I. Congreso Forestal Español, Lorizán (España) 1: 343-350.

VOGT, K.A., R.L. EDMONDS, G.C. ANTOS, D.G. VOGT. 1980. "Relationship between $\mathrm{CO}_{2}$ evolution, ATP concentration and decomposition in four ecosystems in Western Washington", Oikos, 35: 72-79.

WIEDER, R.K., G.E. LANG. 1982. A critique of the analytical methods used in examining decomposition data obtained from litter bags, Ecology 63: 1636-1642.

WITKAMP, M. 1966. "Decomposition of leaf litter in relation to environment microflora respiration", Ecology 47: 194-201. 